\title{
Stimulated Raman Adiabatic Passage in a Dense Medium
}

\author{
B. D. Fainberg ${ }^{1,2}$ and B. Levinsky ${ }^{1}$ \\ ${ }^{1}$ Faculty of Sciences, Holon Institute of Technology, 52 Golomb Steet, Holon 58102, Israel \\ ${ }^{2}$ Raymond and Beverly Sackler Faculty of Exact Sciences, School of Chemistry, Tel-Aviv University, Tel Aviv 69978, Israel
}

Correspondence should be addressed to B. D. Fainberg, fainberg@hit.ac.il

Received 11 September 2010; Accepted 23 October 2010

Academic Editor: Kenneth Ruud

Copyright ( 92010 B. D. Fainberg and B. Levinsky. This is an open access article distributed under the Creative Commons Attribution License, which permits unrestricted use, distribution, and reproduction in any medium, provided the original work is properly cited.

\begin{abstract}
We have considered a coherent population transfer to a higher excited singlet state $\left(S_{2}\right)$ of molecules with anomalous fluorescence in molecular assemblies (e.g., a dense medium). A direct excitation to $S_{2}$ requires light in the UV region. Because of this, the transition is conveniently realized by a two-step (two-photon) process: $S_{0} \rightarrow S_{1} \rightarrow S_{2}$, where transitions $S_{0} \rightarrow S_{1}$ and $S_{1} \rightarrow S_{2}$ correspond to the optical region. We have shown that efficient stimulated Raman adiabatic passage (STIRAP) in the ladder configuration can be realized in this case, using suitably chirped pulses, to compensate a change of the two-photon transition frequency in time, induced by the pulses themselves, due to near dipole-dipole interactions. We have provided a reduced state formulation of the optical control process. Chirping the "pump" pulse that excites transition $S_{0} \rightarrow S_{1}$ is nonequivalent to chirping the "Stokes" pulse that excites transition $S_{1} \rightarrow S_{2}$, with respect to the population of the intermediate state $\left(S_{1}\right)$ in the pulse nonadiabatic regime. We have also shown that with suitably chirped pulses, efficient STIRAP still persists even for a rather large decay of the intermediate state.
\end{abstract}

\section{Introduction}

Population transfer to a higher singlet state $S_{n}(n>1)$ of complex organic molecules makes no sense, since more often than not $S_{n}(n>1)$ rapidly relaxes back to the first excited singlet $S_{1}$ [1-4], though excited-state absorption $S_{1} \rightarrow S_{n}$ $(n>1)$ has a profound effect on coherent population transfer $S_{0} \rightarrow S_{1}$ with shaped laser pulses [5]. The situation reverses for molecules with anomalous fluorescence like azulene, the molecule known as the textbook exception to Kasha's rule [6], and its derivatives. For such molecules, the first excited singlet state $S_{1}$ is very short lived ( $\left.\sim 1 \mathrm{ps}\right)$ due to a conical intersection [7] and, as a consequence, a strong nonradiative decay of the transition $S_{1} \rightarrow S_{0}$. However, a higher excited singlet state $S_{2}$ is long lived $(\sim 1 \mathrm{~ns})$ displaying a strong fluorescence band that corresponds to transition $S_{2} \rightarrow S_{0}$ [8-11]. Because of this, there are also reasons to consider a coherent population transfer to higher excited singlet states $\left(S_{2}\right)$ of the molecules under discussion. However, a direct excitation to a higher electronic state $S_{0} \rightarrow S_{2}$ requires light in the UV region where pulse shaping presents difficulties. Because of this, a coherent population transfer to $S_{2}$ is conveniently realized by a two-step (two-photon) process, $S_{0} \rightarrow S_{1} \rightarrow S_{2}$ where transitions $S_{0} \rightarrow S_{1}$ and $S_{1} \rightarrow$ $S_{2}$ correspond to the optical region. Due to the absence of the center of symmetry in azulene (belongs to the $C_{s}$ point group) and its derivatives, all three transitions $S_{0} \rightarrow$ $S_{2}, S_{0} \rightarrow S_{1}$ and $S_{1} \rightarrow S_{2}$ are allowed. In this regard, using stimulated Raman adiabatic passage (STIRAP) [1216] in the ladder configuration for the population transfer from $S_{0}$ to $S_{2}$ seems rather attractive. Indeed, STIRAP is a simple and powerful technique for complete and robust population transfer in three-state quantum systems. Using this technique, the population is transferred adiabatically from an initially populated state $|1\rangle\left(S_{0}\right)$ to a target state $|3\rangle\left(S_{2}\right)$, which are coupled via an intermediate state $|2\rangle$ $\left(S_{1}\right)$ by two pulsed fields. A unique and very useful feature of STIRAP is that the intermediate state, whose presence is crucial for providing two strongly coupled single-photon transitions, never gets populated, not even transiently. The reason is that throughout the adiabatic evolution of the system, the population remains trapped in an adiabatic dark state, which is a superposition of states $|1\rangle$ and $|3\rangle$ only and does not involve the intermediate state $|2\rangle$ that can be 
a strongly decaying state $\left(S_{1}\right)$. Such a dark state is formed by maintaining a two-photon resonance between the target $|3\rangle$ and initial $|1\rangle$ states during the interaction.

Azulene is a molecular multilevel system. In this relation, it is worthy to note the STIRAP application of controlling non-Franck-Condon transitions in $\mathrm{Na}_{2}$ [17] and adiabatic passage by light-induced potentials in polyatomic molecules [18]. The effect of background states on STIRAP was considered by Kurkal and Rice [19] and Cheng et al. [20]. Nakajima et al. [21] discussed population transfer through continuum intermediate states.

In this study, we will concentrate on another aspect of STIRAP in molecular systems. The point is that many important molecular systems are made out of assemblies of coupled localized chromophores. Examples are molecular crystals, organic nanostructures, supramolecular structures, and so forth, which are of interest from the point of view of the design of new optical materials with desirable characteristics. Therefore, in this paper, we aim to apply STIRAP rather to the assembly of interacting azulene molecules (or its derivatives) than to noninteracting molecules. In general, calculating optical properties of interacting molecules becomes an intractable many-body problem even for a reduced state formulation of the optical control process in an isolated molecule which is used in this work (see below). The local field approximation (see, e.g., [22]) provides a simple phenomenological way of relating the optical characteristics of isolated molecules to those of molecular assemblies. In the local field approximation the corresponding equations of motion, describing the system evolution, become nonlinear thus displaying the dependence of the transition frequencies on optical excitation [23, 24]. Here, we will consider a dense medium of the molecules with the dependence of the transition frequencies on optical excitation due to near dipole-dipole (DD) interactions [25]. In the following, we show that efficient STIRAP in the ladder configuration can be achieved in a dense collection of molecules exhibiting anomalous fluorescence, but only if a change of the twophoton transition frequency, due to near DD interactions, is taken into account.

A two-photon resonance between the initial and final states during the interaction can not be achieved since its frequency depends on the population of these states (manybody effect) (see (13) and (20) below). Our goal is to get an efficient STIRAP in this system. We show that one can use pulses that are suitably chirped (time-dependent carrier frequency) to compensate for a change of frequency of the two-photon transition in time induced by the pulses themselves (a similar idea was proposed in studies of a twostate system in relation to Rabi oscillations in inter-subband transitions in quantum wells [26].)

The outline of the paper is as follows. In Section 2, we introduce our model and derive a closed set of equations for the expectation values of one-particle variables in the local field approximation. In Section 3, we show that suitably chirped pulses that compensate a change of frequency of the two-photon transition in time induced by the pulses themselves enable us to achieve a "dark" state and, hence, complete population transfer in the interacting media. We provide numerical solutions of the coupled equations for the expectation values of one-particle variables in Section 4. We summarize our results in Section 5.

\section{Model and Basic Equations}

Consider a dense collection of molecules, with densities such that there are many molecules within a cubic molecular resonance wavelength, with three electronic states with energies $E_{1}<E_{2}<E_{3}$ under the action of two (phase modulated) pulses $\mathbf{E}_{1}(t)$ and $\mathbf{E}_{2}(t)$

$$
\mathbf{E}(\mathbf{r}, t)=\sum_{i=1,2} \mathbf{E}_{i}(t)=\frac{1}{2} \sum_{i=1,2} \mathbf{e}_{i} \mathcal{E}_{i}(t) \exp \left(-i \omega_{i} t+i \varphi_{i}(t)\right)+\text { c.c. }
$$

The frequencies of which are close to those of the transitions $1 \rightarrow 2$ and $1 \rightarrow 3$, respectively. Here, $\mathcal{E}_{i}(t)$ and $\varphi_{i}(t)$ describe the change of the pulse amplitude and phase in time, $\mathbf{e}_{i}$ are unit polarization vectors, and the instantaneous pulse frequencies are $\omega_{i}(t)=\omega_{i}-d \varphi_{i}(t) / d t$. Three states, 1,2 , and 3 correspond to singlet states $S_{0}, S_{1}$, and $S_{2}$, of the azulene molecule, respectively (Figure 2 ). The potential-energy surfaces of polyatomic molecules are multidimensional, and the number of states related to a transition between initial and final states increases with the number of degrees of freedom of a molecule. For that reason, it is desirable to have a reduced space or a reduced state formulation of the optical control process. We will consider azulene in a Shpol'skii matrix for low temperatures; the $S_{2} \rightarrow S_{1}$ fluorescence spectrum shows very narrow lines [10] (see Figure 1). We assume that field $\mathbf{E}_{2}(t)$ connects states 2 and 3 corresponding to $S_{1}$, with the excitation of one vibrational quantum of the vibration $1558 \mathrm{~cm}^{-1}$ and the vibrationless state of $S_{2}$, respectively (see the second intensive line near $\lambda=810 \mathrm{~nm}$ in Figure 1). Of course, there is one more (less intensive) line corresponding to the vibration $1756 \mathrm{~cm}^{-1}$ near the line related to $1558 \mathrm{~cm}^{-1}$. However, we will consider only one state 2 , representing both vibrationally excited states under discussion in $S_{1}$.

We assume that state 1 represents the vibrationless state of a singlet $S_{0}$ and field $\mathbf{E}_{1}(t)$ excites transition $1 \rightarrow 2$ (see Figure 2), so that the sum of frequencies $\omega_{1}(t)+\omega_{2}(t)$ is close to the frequency of purely electronic transition $S_{0} \rightarrow S_{2}$. In the proposed scheme, the frequencies of transitions $1 \rightarrow 2$ and $2 \rightarrow 3$ are well separated in order to realize STIRAP (in spite of close energies of purely electronic transitions $S_{0} \rightarrow S_{1}$ and $\left.S_{1} \rightarrow S_{2}[7,11]\right)$, though complete and robust population transfer is also possible when each of the two laser pulses interacts with each of the pair of states [27].

The interaction of the dense collection of such systems with electromagnetic fields can be written as

$$
\hat{H}_{\text {int }}=-\sum_{n} \hat{\mathbf{P}}_{n} \cdot \mathbf{E}_{l, n}
$$

where $\mathbf{E}_{l, n}$ is the electric field at the location of the $n$th molecule (local electric field), and $\hat{\mathbf{P}}_{n}$ is its optical 


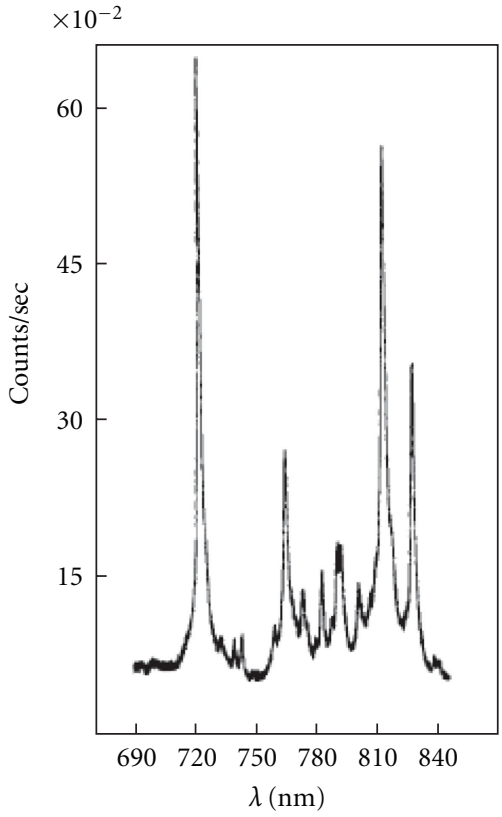

Figure 1: $S_{2} \rightarrow S_{1}$ fluorescence of azulene in $n$-hexane ad $77 \mathrm{~K}$ [10].

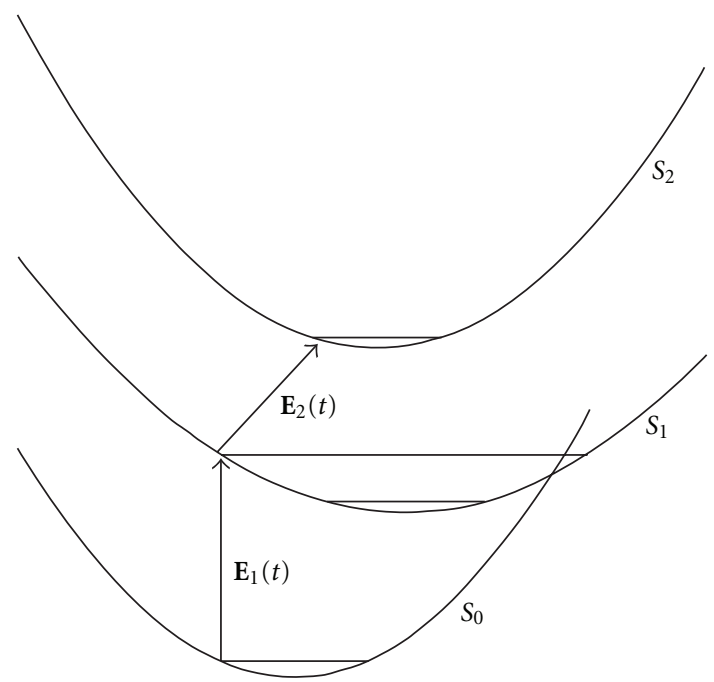

Figure 2: A reduced states formulation of STIRAP in azulene. Field $\mathbf{E}_{2}(t)$ connects states 2 and 3 corresponding to $S_{1}$ with the excitation of one vibrational quantum, and the vibrationless state of $S_{2}$, respectively. State 1 represents the vibrationless state of a singlet $S_{0}$ and field $\mathbf{E}_{1}(t)$ excites transition $1 \rightarrow 2$, so that the sum of frequencies $\omega_{1}(t)+\omega_{2}(t)$ is close to the frequency of purely electronic transition $S_{0} \rightarrow S_{2}$.

polarization operator. The local (microscopic) field is related to the Maxwell (macroscopic) field $\mathbf{E}(\mathbf{r}, t),(1)$, by $[22,25]$

$$
\mathbf{E}_{l, n}=\mathbf{E}(\mathbf{r}, t)+\frac{4 \pi}{3} \sum_{m \neq n} \mathbf{P}_{m},
$$

where $\mathbf{P}_{m}$ denotes the expectation value of $\hat{\mathbf{P}}_{m}$. The second term on the right-hand side of (3) represents the electrostatic longitudinal field created by all other particles $m(\neq n)$, that is, the instantanous dipole-dipole interactions between the molecules.

Let us introduce excitonic operators $\hat{A}_{n, 12} \equiv|n 1\rangle\langle n 2|$, $\widehat{A}_{n, 23} \equiv|n 2\rangle\langle n 3|$ and $\widehat{A}_{n, 13} \equiv|n 1\rangle\langle n 3|$, where $|n k\rangle$ denotes the $k$ state of molecule $n$. Then, the optical polarization operator of the $n$th molecule is given by

$$
\widehat{\mathbf{P}}_{n}=\left(\mathbf{D}_{n, 12} \widehat{A}_{n, 12}^{+}+\mathbf{D}_{n, 23} \widehat{A}_{n, 23}^{+}+\mathbf{D}_{n, 13} \widehat{A}_{n, 13}^{+}\right)+\text {H.c. }
$$

where $\mathbf{D}_{n, k k^{\prime}}$ is the transition dipole moment connecting states $k$ and $k^{\prime}$ of molecule $n$, and H.c. denotes Hermitian conjugate. We also introduce operators $\hat{n}_{m k}=|m k\rangle\langle m k|$ describing the population of states $|m k\rangle$. It is obvious that

$$
\hat{n}_{m 1}+\hat{n}_{m 2}+\hat{n}_{m 3}=I
$$

due to the completeness relation for the 3-level electronic states space of the $m$ th molecule. Here $I$ is the unit operator. Operators $\hat{A}_{n, k k^{\prime}}$ obey the following commutation relation:

$$
\left[\widehat{A}_{m, k k^{\prime}}, \hat{A}_{m, k k^{\prime}}^{+}\right]=\hat{n}_{m k}-\hat{n}_{m k^{\prime}}
$$

where $k=1,2, k^{\prime}=2,3$, and $k<k^{\prime}$. Then, the Hamiltonian of the dense collection of three-state systems under consideration can be written as

$$
\hat{H}=\hat{H}_{0}+\hat{H}_{\text {int }} \text {, }
$$

where

$$
\hat{H}_{0}=\sum_{m} \sum_{k=1,2,3} E_{m k} \hat{n}_{m k} .
$$

$E_{m k}$ is the energy of state $k$ of a molecule $m$.

Using the Heisenberg equations of motion, one obtains the equation for the expectation value of any operator $\hat{F}$

$$
\frac{d}{d t}\langle\hat{F}\rangle=\frac{i}{\hbar}\left\langle\left[\hat{H}_{0}+\hat{H}_{\text {int }}, \hat{F}\right]\right\rangle \equiv \frac{i}{\hbar} \operatorname{Tr}\left(\left[\hat{H}_{0}+\hat{H}_{\text {int }}, \hat{F}\right] \rho\right),
$$

where $\rho$ is the density matrix. Straightforward operator algebra manipulations, using commutation relations, (6), yield equations for $n_{m k} \equiv\left\langle\hat{n}_{m k}\right\rangle$ and $A_{m, k k^{\prime}} \equiv\left\langle\widehat{A}_{m, k k^{\prime}}\right\rangle$ in the rotating wave approximation (RWA). In the derivation of these equations, we kept only single-particle variables, using a factorization into population variables $n_{m k}$ and polarizations $A_{m, k k^{\prime}}$ in accordance with the local field approximation [22]. Then, switching to the system that rotates with instantaneous frequency,

$$
\begin{aligned}
& a_{m, 12}=A_{m, 12} \exp \left[i \omega_{1} t-i \varphi_{1}(t)\right], \\
& a_{m, 23}=A_{m, 23} \exp \left[i \omega_{2} t-i \varphi_{2}(t)\right], \\
& a_{m, 13}=A_{m, 13} \exp \left[i\left(\omega_{1}+\omega_{2}\right) t-i\left(\varphi_{1}(t)+\varphi_{2}(t)\right)\right],
\end{aligned}
$$

we obtain equations for the quantities that vary slowly with time during the period of a light wave. Furthermore, considering a homogeneous excitation of an assembly of identical 
molecules $\left(\mathbf{D}_{n, k k^{\prime}}=\mathbf{D}_{k k^{\prime}}, E_{m k}=E_{k}, n_{m k}=n_{k}, a_{m, k k^{\prime}}=a_{k k^{\prime}}\right)$, we get

$$
\begin{aligned}
\frac{d a_{12}}{d t}=-i & \left\{\left[\omega_{21}-\omega_{1}(t)+p_{12}\left(n_{2}-n_{1}\right)\right] a_{12}+\frac{\Omega_{1}(t)}{2}\right. \\
& \left.\times\left(n_{2}-n_{1}\right)-\frac{\Omega_{2}(t)}{2} a_{13}+\left(p_{13}-p_{23}\right) a_{23}^{*} a_{13}\right\},
\end{aligned}
$$

$$
\begin{gathered}
\frac{d a_{23}}{d t}=-i\left\{\left[\omega_{32}-\omega_{2}(t)+p_{23}\left(n_{3}-n_{2}\right)\right] a_{23}+\frac{\Omega_{1}(t)}{2} a_{13}\right. \\
\left.+\frac{\Omega_{2}(t)}{2}\left(n_{3}-n_{2}\right)+\left(p_{12}-p_{13}\right) a_{12}^{*} a_{13}\right\},
\end{gathered}
$$

$$
\begin{aligned}
\frac{d a_{13}}{d t}=-i\{[ & \left.\omega_{31}-\omega_{1}(t)-\omega_{2}(t)+p_{13}\left(n_{3}-n_{1}\right)\right] a_{13} \\
& \left.+\frac{\Omega_{1}(t)}{2} a_{23}-\frac{\Omega_{2}(t)}{2} a_{12}+\left(p_{12}-p_{23}\right) a_{23} a_{12}\right\},
\end{aligned}
$$

$$
\begin{aligned}
& \frac{d}{d t}\left(\begin{array}{l}
n_{1} \\
n_{3}
\end{array}\right)=\left(\begin{array}{c}
-\Omega_{1}(t) \operatorname{Im} a_{12} \\
\Omega_{2}(t) \operatorname{Im} a_{23}
\end{array}\right), \\
& n_{1}+n_{2}+n_{3}=1,
\end{aligned}
$$

where $\omega_{k k^{\prime}}=\left(E_{k}-E_{k^{\prime}}\right) / \hbar$ and $\Omega_{i}(t)=\left(\mathbf{D}_{i, i+1} \cdot \mathbf{e}_{i}\right) \mathcal{E}_{i}(t) / \hbar$ is the Rabi frequency corresponding to the $i$ th pulse. The strength of the near DD interaction is given by terms

$$
p_{k k^{\prime}}=\frac{4 \pi}{3 \hbar}\left|D_{k k^{\prime}}\right|^{2} N,
$$

where $N$ is the density of molecules [25]. In writing (11)(14), we have neglected the population-decay time of state 2 $\left(S_{1}\right)$. Extending this consideration to a decaying intermediate state is made in Section 4.2.

One can easily see from (13) that the two-photon resonance cannot be achieved with the pulses of fixed frequency, at least due to the presence of the "local field" term $p_{13}\left(n_{3}-\right.$ $n_{1}$ ) that depends on the state populations. However, suitably chirped pulses can compensate the "local field" detuning $p_{13}\left(n_{3}-n_{1}\right)$, and the first term on the right-hand side of (13) may be zero during the interaction. We will address the question of whether the chirping allows the realisation of a "dark" state and thus allows complete population transfer in the interacting media in the next section.

\section{3. "Dark" State in the Interacting Media}

The expectation values $a_{m, k k^{\prime}}$ in the system that rotates with instantaneous frequency, (10), can be expressed through the density matrix. Indeed,

$$
\begin{aligned}
a_{m, k k^{\prime}} & =\operatorname{Tr}\left(\hat{a}_{m, k k^{\prime}} \rho\right) \\
& =\operatorname{Tr}\left(|m k\rangle\left\langle m k^{\prime}\left|\sum_{j j^{\prime}}\right| m j\right\rangle \rho_{m, j j^{\prime}}\left\langle m j^{\prime}\right|\right)=\rho_{m, k^{\prime} k} .
\end{aligned}
$$

In particular, for pure states that are realized in the absence of relaxation, the density matrix can be represented as $\rho_{k^{\prime} k}=c_{k}^{*} c_{k^{\prime}}$ where $c_{k}$ are the amplitudes of states, and we consider a homogeneous excitation of an assembly of identical molecules. This enables us to express $a_{k k^{\prime}}$ by the amplitudes of states $c_{k}$. Putting $a_{k k^{\prime}}=c_{k}^{*} c_{k^{\prime}}$ in equations (11)-(15), we get the following equations for the magnitudes $c_{k}$ :

$$
\begin{gathered}
\frac{d c_{1}}{d t}=i \frac{\Omega_{1}(t)}{2} c_{2} \\
\frac{d c_{2}}{d t}=-i\left[\omega_{21}-\omega_{1}(t)+p_{12}\left(\left|c_{2}\right|^{2}-\left|c_{1}\right|^{2}\right)+\left(p_{13}-p_{23}\right)\left|c_{3}\right|^{2}\right] c_{2} \\
+i \frac{\Omega_{1}(t)}{2} c_{1}+i \frac{\Omega_{2}(t)}{2} c_{3} \\
\frac{d c_{3}}{d t}=-i\left[\omega_{31}-\omega_{1}(t)-\omega_{2}(t)+p_{13}\left(\left|c_{3}\right|^{2}-\left|c_{1}\right|^{2}\right)\right. \\
\left.+\left(p_{12}-p_{23}\right)\left|c_{2}\right|^{2}\right] c_{3}+i \frac{\Omega_{2}(t)}{2} c_{2} \\
\left|c_{1}\right|^{2}+\left|c_{2}\right|^{2}+\left|c_{3}\right|^{2}=1
\end{gathered}
$$

Let us assume that pulses $\mathbf{E}_{1}(t)$ and $\mathbf{E}_{2}(t)$ are suitably chirped to fulfill the condition of the two-photon resonance during the interaction (the first term on the right-hand side of (20) is equal to zero). Then, one can easily see that under the adiabatic conditions $\left(d c_{k} / d t=0\right)$, the solutions of (18)(21) are given by $c_{2}=0, c_{3}=-\left[\Omega_{1}(t) / \Omega_{2}(t)\right] c_{1}$ and

$$
c_{1}=\frac{\Omega_{2}(t)}{\sqrt{\Omega_{1}^{2}(t)+\Omega_{2}^{2}(t)}} .
$$

This enables us to obtain the adiabatic "dark" state $\Psi_{\text {ad }}=$ $c_{1}|1\rangle+c_{3}|3\rangle$ as a superposition of states $|1\rangle$ and $|3\rangle$ only, similar to the case of usual noninteracting media without local field effects [12]. Complete population transfer occurs if $\lim _{t \rightarrow-\infty}\left[\Omega_{1}(t) / \Omega_{2}(t)\right]=0$ and $\lim _{t \rightarrow+\infty}\left[\Omega_{2}(t) / \Omega_{1}(t)\right]=0$, where $t \rightarrow-\infty$ and $t \rightarrow+\infty$ correspond to times before and after the interaction with optical pulses, respectively.

\section{Numerical Calculations}

4.1. Calculations without Decay. To check these ideas, we provided numerical solutions of coupled equations (11)(15). For this purpose, it is convenient to switch to equations for the components of the 3-state coherence vector [28]

$$
\begin{gathered}
v_{k k^{\prime}}=i\left(a_{k k^{\prime}}-a_{k^{\prime} k}\right), \quad u_{k k^{\prime}}=a_{k k^{\prime}}+a_{k^{\prime} k}, \\
w_{1}=n_{2}-n_{1}, \quad w_{2}=-\sqrt{\frac{1}{3}}\left(n_{2}+n_{1}-2 n_{3}\right),
\end{gathered}
$$


where $k, k^{\prime}=1,2,3$ and $k<k^{\prime}$. These components satisfy the following equations:

$$
\begin{aligned}
& \frac{d u_{12}}{d t}=-\Delta_{21} v_{12}+\frac{\Omega_{2}}{2} v_{13}, \\
& \frac{d u_{23}}{d t}=-\Delta_{32} v_{23}-\frac{\Omega_{1}}{2} v_{13}, \\
& \frac{d u_{13}}{d t}=-\Delta_{31} v_{13}-\frac{\Omega_{1}}{2} v_{23}+\frac{\Omega_{2}}{2} v_{12}, \\
& \frac{d v_{12}}{d t}=\Delta_{21} u_{12}+\Omega_{1} w_{1}-\frac{\Omega_{2}}{2} u_{13}, \\
& \frac{d v_{23}}{d t}=\Delta_{32} u_{23}+\frac{\Omega_{1}}{2} u_{13}+\frac{\Omega_{2}}{2}\left(\sqrt{3} w_{2}-w_{1}\right), \\
& \frac{d v_{13}}{d t}=\Delta_{31} u_{13}+\frac{\Omega_{1}}{2} u_{23}-\frac{\Omega_{2}}{2} u_{12}, \\
& \frac{d w_{1}}{d t}=-\Omega_{1} v_{12}+\frac{\Omega_{2}}{2} v_{23}, \\
& \frac{d w_{2}}{d t}=-\sqrt{3} \Omega_{2} v_{23},
\end{aligned}
$$

where detunings with respect to the renormalized optical transitions are given by

$$
\begin{aligned}
\Delta_{21}= & \omega_{21}-\omega_{1}(t)+p_{12} w_{1}+\left(p_{13}-p_{23}\right)\left(\frac{\sqrt{3}}{3} w_{2}+\frac{1}{3}\right) \\
\Delta_{32}= & \omega_{32}-\omega_{2}(t)+\frac{\sqrt{3}}{2} w_{2}\left[p_{23}-\frac{1}{3}\left(p_{12}-p_{13}\right)\right] \\
& -\frac{1}{2} w_{1}\left(p_{23}+p_{12}-p_{13}\right)+\frac{1}{3}\left(p_{12}-p_{13}\right), \\
\Delta_{31}= & \omega_{31}-\omega_{1}(t)-\omega_{2}(t)+\frac{\sqrt{3}}{2} w_{2}\left[p_{13}-\frac{1}{3}\left(p_{12}-p_{23}\right)\right] \\
& +\frac{1}{2} w_{1}\left(p_{13}+p_{12}-p_{23}\right)+\frac{1}{3}\left(p_{12}-p_{23}\right) .
\end{aligned}
$$

In deriving equations (24), we have used the relation $a_{k k^{\prime}}=$ $c_{k}^{*} c_{k^{\prime}}$ for pure states.

We considered the excitation of a dense collection of three-state systems with parameters $p_{k k^{\prime}}$, (16), corresponding to those of azulene with two Gaussian pulses of the same duration, $\varepsilon_{i}(t)=\varepsilon_{0 i} \exp \left[-\left(t-t_{i}\right)^{2} /\left(2 \tau^{2}\right)\right]$, and the amplitudes giving the same maximum Rabi frequencies for transitions $1 \rightarrow 2$ and $2 \rightarrow 3$, ordered counterintuitively, $\mathbf{E}_{2}(t)$ before $\mathbf{E}_{1}(t)$. Parameters $p_{k k^{\prime}}$, (16), can be evaluated through radiation decay times $\tau_{\text {rad }}$ of transitions $S_{i} \rightarrow S_{j}$ by $p_{k k^{\prime}}=\varkappa_{k k^{\prime}} N$, where $\varkappa_{k k^{\prime}} \equiv 1 /\left(8 \pi^{2} \omega_{k^{\prime} k}^{3} \tau_{\text {rad }}\right)$, and $\tau_{\text {rad }}$ were taken from [8]: $\tau_{\text {rad }}\left(S_{2} \rightarrow S_{0}\right)=5 \cdot 10^{-9} \mathrm{~s}, \tau_{\mathrm{rad}}\left(S_{1} \rightarrow S_{0}\right)=$ $8 \cdot 10^{-7} \mathrm{~s}$ and $\tau_{\mathrm{rad}}\left(S_{2} \rightarrow S_{1}\right)=2 \cdot 10^{-6} \mathrm{~s}$. This gives $\varkappa_{13}=$ $1.06 \cdot 10^{-7}\left[\mathrm{~cm}^{3} / \mathrm{s}\right], p_{13} \equiv p, p_{12}=0.05 p, p_{23}=0.02 p$. The time delay between the pulses was $t_{1}-t_{2}=2 \tau$, and the pulse areas $S=\int \Omega_{1,2}(t) d t=\sqrt{2 \pi} \Omega_{\max } \tau$ ranged from 2 to 122 . The product of the strength of the near DD interaction, $p$, and parameter $\tau$ characterizing the pulse duration, was equal to $p \tau=20$. This value can be realized, for example, for the density of the active molecules $N=3 \cdot 10^{19} \mathrm{~cm}^{-3}$ and $\tau=6.3 \mathrm{ps}$ (this value of $\tau$ exceeds the decay time of the intermediate state that will be taken into account in Section 4.2). It is worthy to note that $N=3 \cdot 10^{19} \mathrm{~cm}^{-3}$ corresponds to the mean distance between active molecules of $3.2 \mathrm{~nm}$ justifying the use of the dipole approximation for the near DD interaction.

One can see from (20) that the frequency of transition $1 \rightarrow 3$ shifts to the blue side during the population transfer $1 \rightarrow 3$, since $p_{13}>0$ and $p_{13} \gg p_{12}, p_{23}$ for azulene. This denotes that the chirp preserving the two-photon resonance will not bring other levels into play close to the purely electronic transition $S_{0} \rightarrow S_{2}$.

Figure 3 shows the state populations, after the completion of the pulses action as functions of the ratio between the Rabi frequency at the pulse maximum and parameter $p$ for $\omega_{21}-\omega_{1}=0$. Dotted lines correspond to the pulses with fixed frequencies when $\omega_{31}-\left(\omega_{1}+\omega_{2}\right)=0$. Other lines describe the population transfer under the two-photon resonance conditions, achieved by chirping the pulse $\mathbf{E}_{1}(t)$ (solid lines) and $\mathbf{E}_{2}(t)$ (dashed lines). Note that pulse chirping enables us to realize the two-photon resonance during the interaction, giving rise to efficient and robust population transfer by STIRAP in interacting media, in contrast to the excitation with pulses of fixed frequencies. Pronounced oscillations of the populations in the latter case (dotted lines) can be related to bistable (or multistable) behavior of the solution of (24), due to the dependence of the resonance frequency on the population difference $[14,23]$. The bistability effect consists of a sudden switching of the population from a low level to a higher level with increasing pump intensity.

Chirping the pulse $\mathbf{E}_{2}(t)$ gives rise to a much larger population of intermediate state 2 for small values of $\Omega_{\max } / p$, than chirping the pulse $\mathbf{E}_{1}(t)$. This can be explained as follows. Let us assume that the chirped pulse is $\mathbf{E}_{1}(t)$. To obey the two-photon resonance condition (see (20) and (27)), frequency $\omega_{1}(t)$ must be equal to

$$
\omega_{1}(t) \approx \omega_{31}-\omega_{2}+p\left(n_{3}-n_{1}\right)=\omega_{1}+p\left(n_{3}-n_{1}\right),
$$

since for azulene $p_{13} \gg p_{12}, p_{23}$. Substituting $\omega_{1}(t)$ from (28) into (25) for the detuning with respect to the renormalized frequency of transition $2 \rightarrow 1$ (see also (19)), we get $\omega_{21}-$ $\omega_{1}(t)+p n_{3}=\omega_{21}-\omega_{1}+p n_{1}=p n_{1}$. In other words, detuning of transition $2 \rightarrow 1$ with respect to $\omega_{1}(t)$ comprises $p n_{1}$ which prevents populating state 2 at the beginning of the process when $n_{1}$ is close to 1 . In contrast, when one chirps the pulse $\mathbf{E}_{2}(t)$, detuning of transition $2 \rightarrow 1$ with respect to $\omega_{1}$ comprises $\omega_{21}-\omega_{1}+p n_{3}=p n_{3}$ which can be close to zero at the beginning of the process. Therefore, better conditions for populating state 2 are realized when the chirped pulse is $\mathbf{E}_{2}(t)$.

Figure 4 depicts populations $n_{3}$ and $n_{2}$ for the pulses with fixed frequencies when $\omega_{31}-\left(\omega_{1}+\omega_{2}\right)-p_{13}=0$ corresponding to the two-photon resonance condition for the initial populations of states $\left(n_{1}(-\infty)=1\right.$, see $\left.(28)\right)$. 


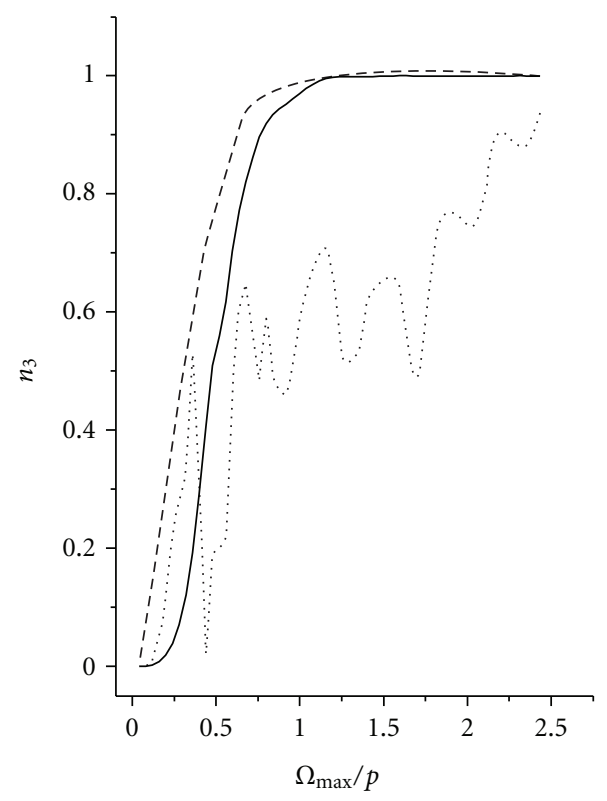

(a)

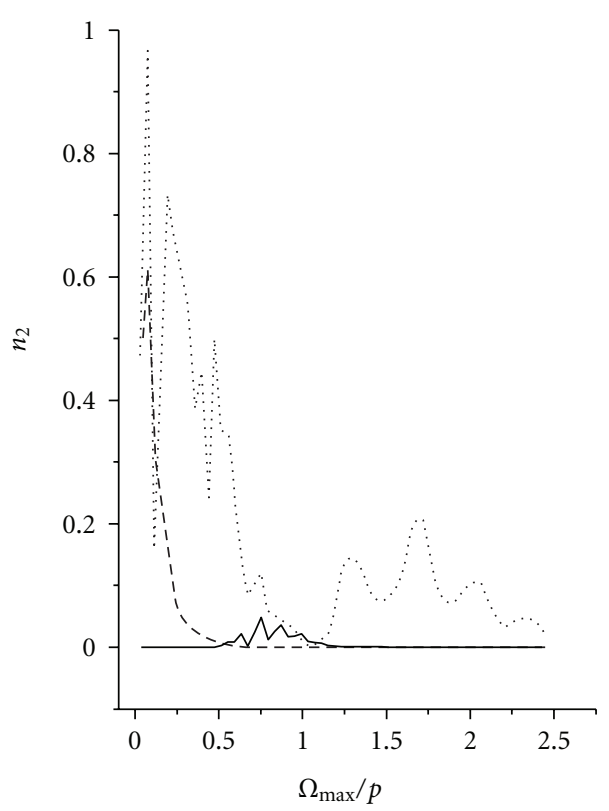

(b)

FIgURe 3: Populations of the final (a) and intermediate (b) states after the completion of the pulses action as functions of $\Omega_{\text {max }} / p$. Solid lines: chirping the pulse $\mathbf{E}_{1}(t)$, dashed: chirping $\mathbf{E}_{2}(t)$, dots: pulses with fixed frequencies for $\omega_{31}-\left(\omega_{1}+\omega_{2}\right)=0$.

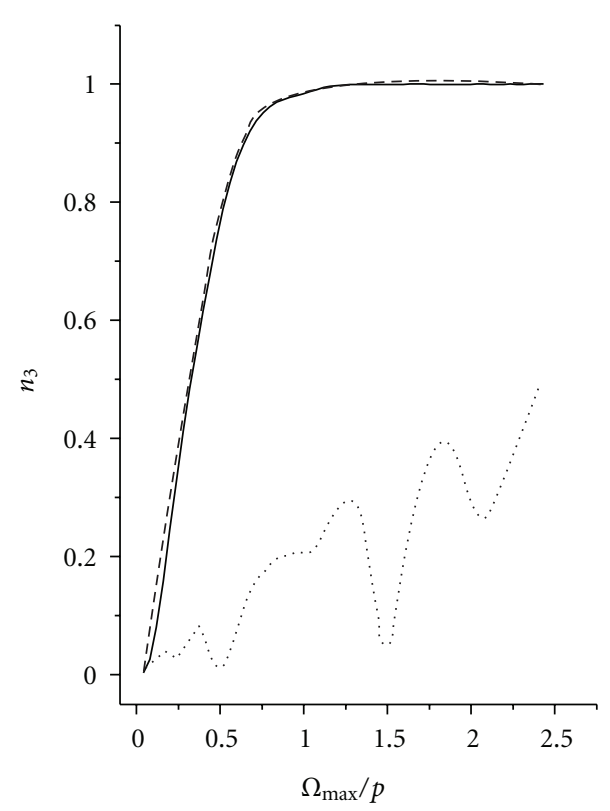

(a)

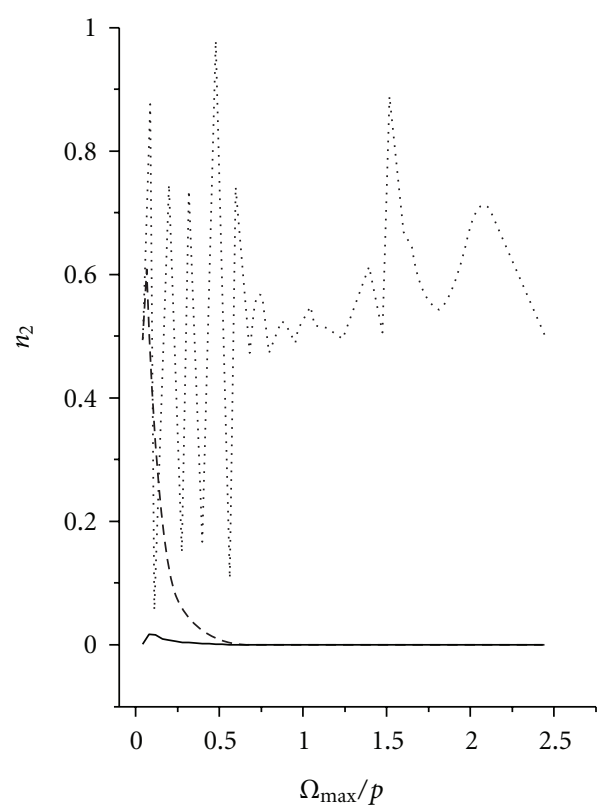

(b)

FIgURE 4: Populations of the final (a) and intermediate (b) states after the completion of the pulses action as functions of $\Omega_{\max } / p$. Solid lines: chirping the pulse $\mathbf{E}_{1}(t)$, dashed: chirping $\mathbf{E}_{2}(t)$, dots: pulses with fixed frequencies for $\omega_{31}-\left(\omega_{1}+\omega_{2}\right)-p_{13}=0$.

Note again that only the pulse chirping gives rise to efficient and robust population transfer by STIRAP in interacting media. As to the excitation with pulses of fixed frequencies, pronounced oscillations remain for both populations $n_{2}$ and $n_{3}$, though $n_{3}$ is essentially smaller than that in Figure 3.
4.2. Influence of the Decay of Intermediate State. In Section 4.1, we have shown that efficient STIRAP for population transfer to higher electronic states in interacting media of molecules with anomalous fluorescence can be realized in the absence of decay, using suitably chirped pulses. However, an intermediate state in this process belongs to 


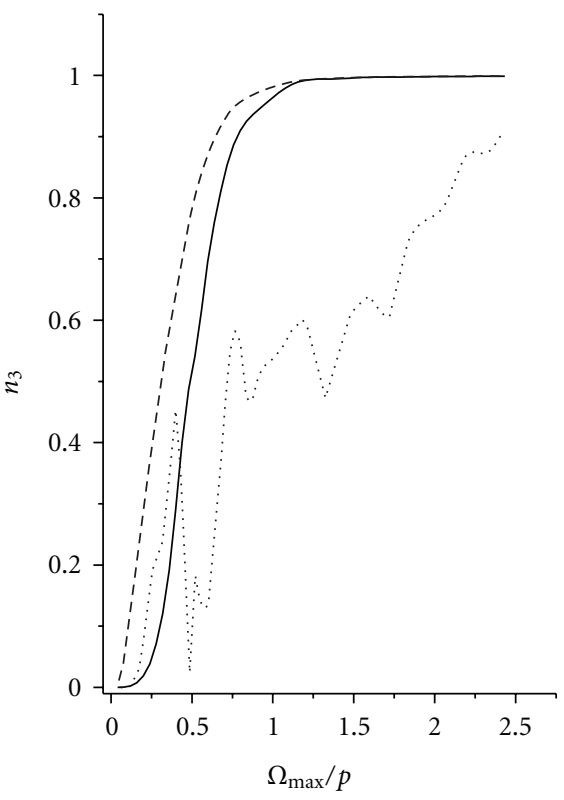

Figure 5: Populations of the final state $n_{3}$ with the decaying intermediate state $(\tau \Gamma=0.6)$ after the completion of the pulses action as functions of $\Omega_{\max } / p$. Solid line: chirping the pulse $\mathbf{E}_{1}(t)$, dashed: chirping $\mathbf{E}_{2}(t)$, dots: pulses with fixed frequencies for $\omega_{31}-$ $\left(\omega_{1}+\omega_{2}\right)=0$. Other parameters are identical to those of Figure 3 .

the first singlet $S_{1}$ which decays very fast into $S_{0}$. In this section, we will extend the above consideration to a decaying intermediate state. We will use (18)-(21) for the magnitudes $c_{k}$ where (19) is replaced by (29)

$$
\begin{aligned}
\frac{d c_{2}}{d t}=-\frac{1}{2} \Gamma c_{2}-i[ & \omega_{21}-\omega_{1}(t)+p_{12}\left(\left|c_{2}\right|^{2}-\left|c_{1}\right|^{2}\right) \\
& \left.+\left(p_{13}-p_{23}\right)\left|c_{3}\right|^{2}\right] c_{2}+i \frac{\Omega_{1}(t)}{2} c_{1}+i \frac{\Omega_{2}(t)}{2} c_{3}
\end{aligned}
$$

taking into account a decaying intermediate state (the first term on the right-hand side of (29)).

We provided numerical solutions of coupled equations (18), (20), (21), and (29). Figures 5 and 6 show that with suitably chirped pulses, the efficient STIRAP still persists even for rather large decay $\tau \Gamma=6$ corresponding to $\tau=6 \mathrm{ps}$ (see Section 4.1) and $1 / \Gamma=1$ ps. However, for the excitation with pulses of fixed frequencies, the oscillations of $n_{3}$ are smoothed at a large decay which may be related to vanishing the bistable (or multistable) behavior for this condition.

\section{Conclusion}

In this work, we have considered a coherent population transfer to a higher excited singlet state $\left(S_{2}\right)$ in molecular assemblies (e.g., a dense medium) of molecules with anomalous fluorescence. A direct excitation to $S_{2}$ requires light in the UV region. Because of this, the transition is conveniently realized by a two-step (two-photon) process: $S_{0} \rightarrow S_{1} \rightarrow$ $S_{2}$ where transitions $S_{0} \rightarrow S_{1}$ and $S_{1} \rightarrow S_{2}$ correspond

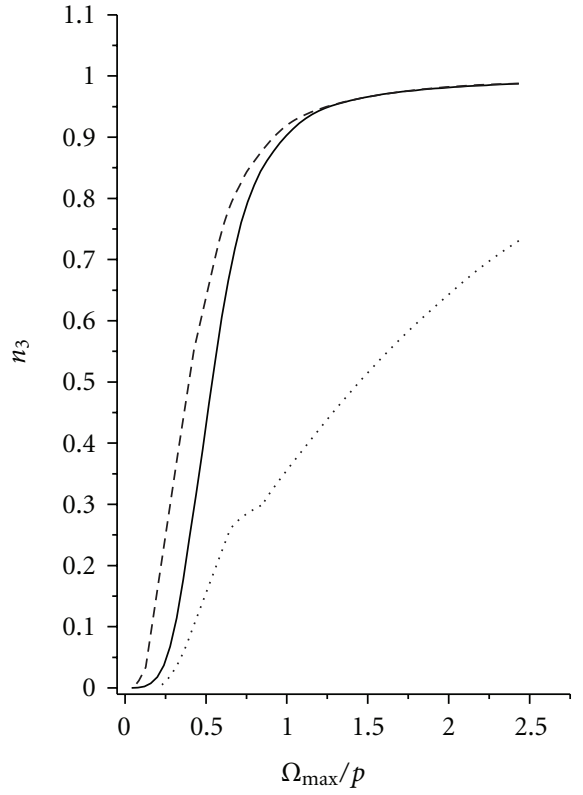

FIgURE 6: The same as in Figure 5 only for $\tau \Gamma=6$.

to the optical region. Since for such molecules, the first excited singlet state $S_{1}$ is very short living, using STIRAP in the ladder configuration for the population transfer $S_{0} \rightarrow$ $S_{2}$ seems rather attractive, since a unique and very useful feature of STIRAP is that the intermediate state $\left(S_{1}\right)$ never gets populated, not even transiently. This is provided by maintaining a two-photon resonance between the target $\left(S_{2}\right)$ and initial $\left(S_{0}\right)$ states during the interaction.

The potential energy surfaces of polyatomic molecules are multidimensional, and the number of states related to a transition between initial and final states increases with the number of degrees of freedom of a molecule. We have provided a reduced states formulation of the optical control process, using azulene in a Shpol'skii matrix for low temperatures as an example.

Unfortunately, in molecular assemblies (a dense medium of molecules) a change of the frequency of the two-photon transition exists due to near DD interactions. We have shown that in spite of the frequency change, efficient STIRAP can be realized in this case as well, using suitably chirped pulses to compensate a change of the two-photon transition frequency in time induced by the pulses themselves. Chirping the "pump" pulse that excites transition $S_{0} \rightarrow S_{1}$ and the "Stokes" pulse that excites transition $S_{1} \rightarrow S_{2}$ are nonequivalent with respect to the population of the intermediate state $\left(S_{1}\right)$ in the pulse nonadiabatic regime. We have also shown that with suitably chirped pulses, the efficient STIRAP still persists, even for a rather large decay of the intermediate state.

It is worthy to note that the method for the compensation of two-photon detuning, proposed in our work, can be used not only in the case of violating the two-photon resonance due to near DD interactions, but also due to the off-resonant Stark shift [29]. In general, the pulse chirp must compensate the two-photon detuning stemming from both cases. 


\section{Acknowledgment}

This work was supported by a grant from the Russia-Israel Scientific Research Cooperation.

\section{References}

[1] V. L. Bogdanov and V. P. Klochkov, "Hot luminescence and vibrational relaxation in complex molecules with diffuse spectra," Optics and Spectroscopy, vol. 44, no. 4, pp. 412-416, 1978.

[2] V. L. Bogdanov and V. P. Klochkov, "Luminescence from higher excited states and vibrational relaxation of polyatomic molecules in solutions," Optics and Spectroscopy, vol. 45, pp. 51-54, 1978.

[3] V. L. Bogdanov and V. P. Klochkov, "Effect of temperature and medium on secondary luminescence of 1,2-benzanthracene during excitation of higher electronic states," Optics and Spectroscopy, vol. 52, no. 1, pp. 41-45, 1982.

[4] S. A. Kovalenko, J. Ruthmann, and N. P. Ernsting, "Ultrafast stokes shift and excited-state transient absorption of coumarin 153 in solution," Chemical Physics Letters, vol. 271, no. 1-3, pp. 40-50, 1997.

[5] B. D. Fainberg and V. A. Gorbunov, "Adiabatic passage in a three-state system with non-Markovian relaxation: the role of excited-state absorption and two-exciton processes," Journal of Physical Chemistry A, vol. 111, no. 38, pp. 9560-9569, 2007.

[6] M. Kasha, "Characterization of electronic transitions in complex molecules," Discussions of the Faraday Society, vol. 9, pp. 14-19, 1950.

[7] M. J. Bearpark, F. Bernardi, S. Clifford et al., "The azulene S1 state decays via a conical intersection: a CASSCF study with MMVB dynamics," Journal of the American Chemical Society, vol. 118, no. 1, pp. 169-175, 1996.

[8] D. Huppert, J. Jortner, and P. M. Rentzepis, "Laser excited emission spectroscopy of azulene in the gas phase," The Journal of Chemical Physics, vol. 56, no. 10, pp. 4826-4833, 1972.

[9] J. M. Friedman and R. M. Hochstrasser, "Moderately high resolution fluorecence spectrum of the S1 $\rightarrow$ S0 transition of azulene," Chemical Physics, vol. 6, no. 2, pp. 145-154, 1974.

[10] G. D. Gillispie and E. C. Lim, "S2 $\rightarrow$ S1 fluorescence of azulene in a Shpol'skii matrix," The Journal of Chemical Physics, vol. 65, no. 10, pp. 4314-4316, 1976.

[11] M. Dierksen and S. Grimme, "Density functional calculations of the vibronic structure of electronic absorption spectra," Journal of Chemical Physics, vol. 120, no. 8, pp. 3544-3554, 2004.

[12] K. Bergmann, H. Theuer, and B. W. Shore, "Coherent population transfer among quantum states of atoms and molecules," Reviews of Modern Physics, vol. 70, no. 3, pp. 1003-1025, 1998.

[13] N. V. Vitanov, T. Halfmann, B. W. Shore, and K. Bergmann, "Laser-induced population transfer by adiabatic passage techniques," Annual Review of Physical Chemistry, vol. 52, pp. 763-809, 2001.

[14] E. M. Graefe, H. J. Korsch, and D. Witthaut, "Mean-field dynamics of a Bose-Einstein condensate in a time-dependent triple-well trap: nonlinear eigenstates, Landau-Zener models, and stimulated Raman adiabatic passage," Physical Review A, vol. 73, no. 1, Article ID 013617, 2006.

[15] A. A. Rangelov, N. V. Vitanov, L. P. Yatsenko, B. W. Shore, T. Halfmann, and K. Bergmann, "Stark-shift-chirped rapidadiabatic-passage technique among three states," Physical Review A, vol. 72, no. 5, Article ID 053403, 2005.
[16] E. A. Shapiro, A. Pe'er, J. Ye, and M. Shapiro, "Piecewise adiabatic population transfer in a molecule via a wave packet," Physical Review Letters, vol. 101, no. 2, Article ID 023601, 2008.

[17] V. S. Malinovsky, J. Santamaría, and I. R. Solá, "Controlling non-Franck-condon transitions: counterintuitive schemes of population transfer in the adiabatic and strong adiabatic regimes," Journal of Physical Chemistry A, vol. 107, no. 40, pp. 8259-8270, 2003.

[18] J. González-Vázquez, I. R. Sola, and J. Santamaría, "Adiabatic passage by light-induced potentials in polyatomic molecules," Journal of Physical Chemistry A, vol. 110, no. 4, pp. 1586-1593, 2006.

[19] V. Kurkal and S. A. Rice, "Sensitivity of the extended STIRAP method of selective population transfer to coupling to background states," Journal of Physical Chemistry B, vol. 105, no. 28, pp. 6488-6494, 2001.

[20] T. Cheng, H. Darmawan, and A. Brown, "Stimulated Raman adiabatic passage in molecules: the effects of background states," Physical Review A, vol. 75, no. 1, Article ID 013411, 2007.

[21] T. Nakajima, M. Elk, J. Zhang, and P. Lambropoulos, "Population transfer through the continuum," Physical Review A, vol. 50, no. 2, pp. R913-R916, 1994.

[22] S. Mukamel, Principles of Nonlinear Optical Spectroscopy, Oxford University Press, New York, NY, USA, 1995.

[23] V. Malyshev and P. Moreno, "Mirrorless optical bistability of linear molecular aggregates," Physical Review A, vol. 53, no. 1, pp. 416-423, 1996.

[24] G. Y. Slepyan, A. Magyarov, S. A. Maksimenko, A. Hoffmann, and D. Bimberg, "Rabi oscillations in a semiconductor quantum dot: influence of local fields," Physical Review B, vol. 70, no. 4, Article ID 045320, 2004.

[25] M. E. Crenshaw, M. Scalora, and C. M. Bowden, "Ultrafast intrinsic optical switching in a dense medium of two-level atoms," Physical Review Letters, vol. 68, no. 7, pp. 911-914, 1992.

[26] A. A. Batista and D. S. Citrin, "Rabi flopping in a two-level system with a time-dependent energy renormalization: intersubband transitions in quantum wells," Physical Review Letters, vol. 92, no. 12, Article ID 127404, 2004.

[27] R. Unanyan, S. Guérin, B. W. Shore, and K. Bergmann, "Efficient population transfer by delayed pulses despite coupling ambiguity," European Physical Journal D, vol. 8, no. 3, pp. 443-449, 2000.

[28] F. T. Hioe and J. H. Eberly, "N-level coherence vector and higher conservation laws in quantum optics and quantum mechanics," Physical Review Letters, vol. 47, no. 12, pp. 838-841, 1981.

[29] S. Guérin, L. P. Yatsenko, T. Halfmann, B. W. Shore, and K. Bergmann, "Stimulated hyper-Raman adiabatic passage, II. Static compensation of dynamic Stark shifts," Physical Review A, vol. 58, no. 6, pp. 4691-4704, 1998. 


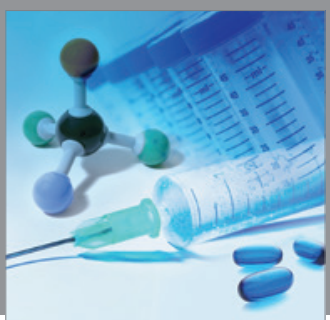

International Journal of

Medicinal Chemistry

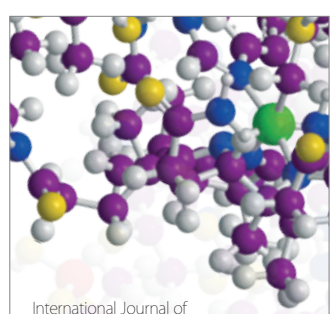

Carbohydrate Chemistry

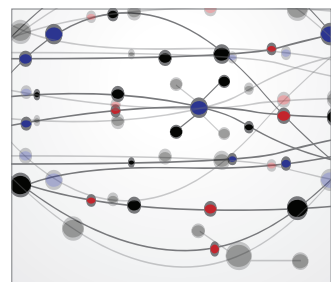

The Scientific World Journal
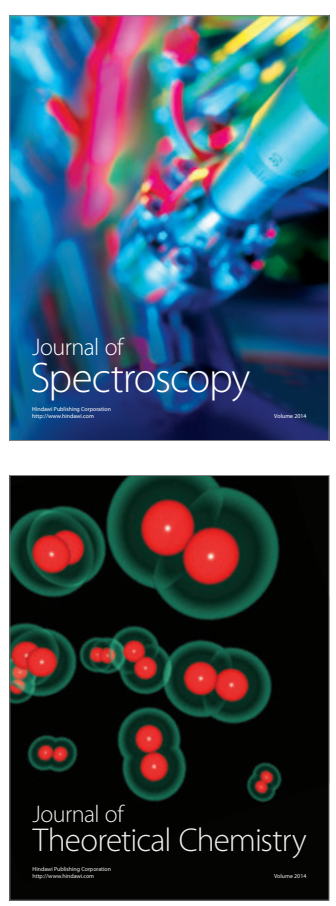
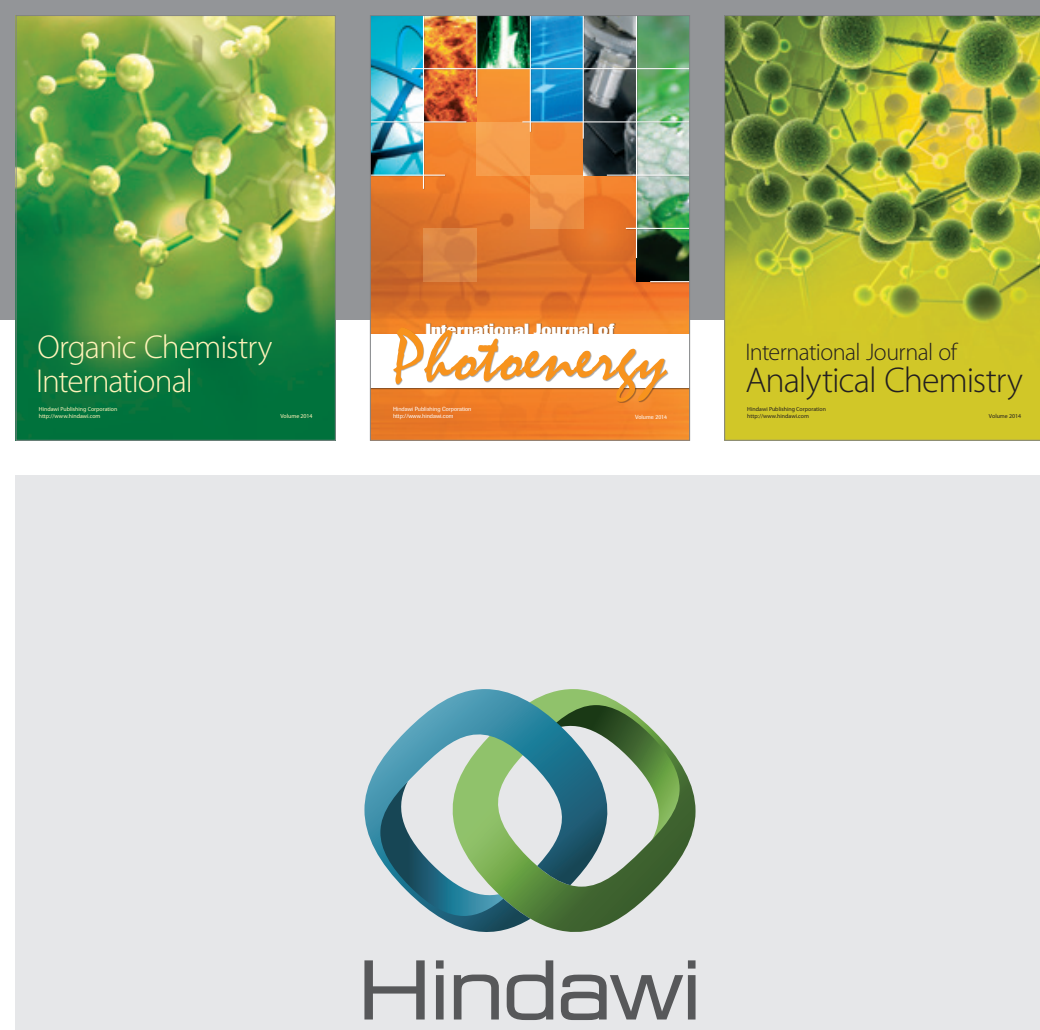

Submit your manuscripts at

http://www.hindawi.com
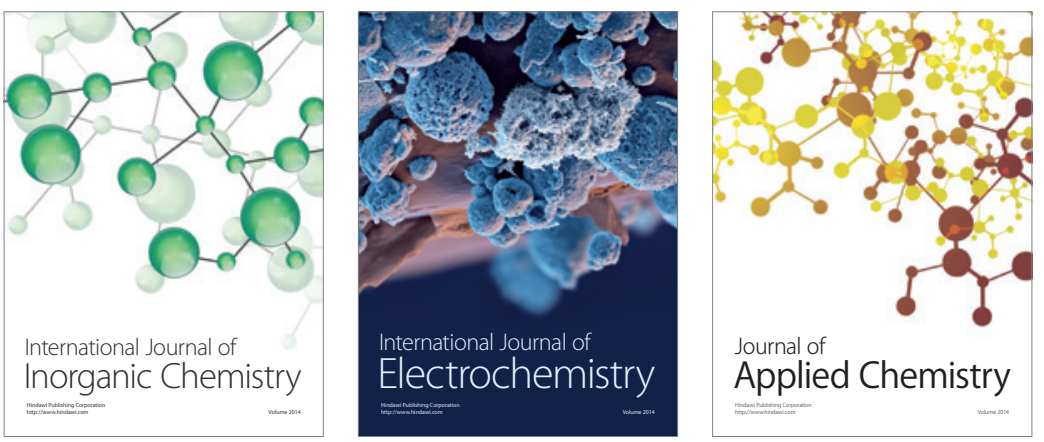

Journal of

Applied Chemistry
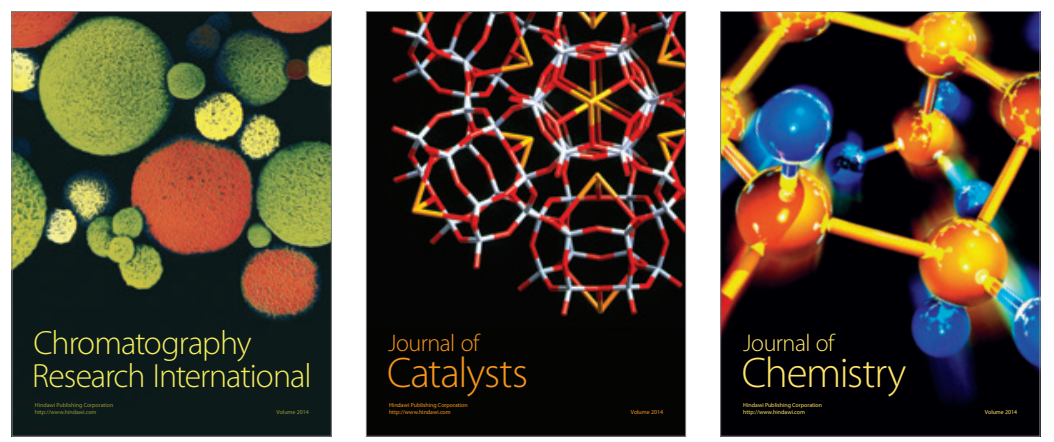
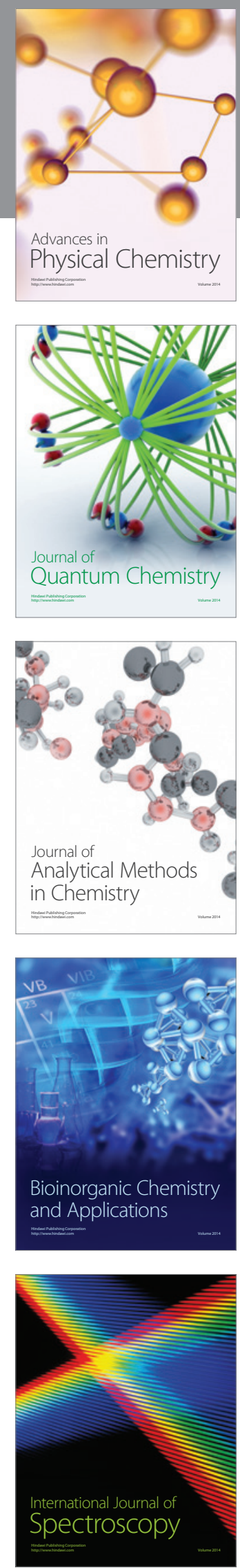